\title{
Estado actual de la prostatectomía radical laparoscópica
}

\author{
Cecchini Rosell L, Esquena Fernández S, Raventós Busquets CX, Orsola de los Santos A. \\ Trilla Herrera E, Planas Morin J, Morote Robles J
}

Servicio de Urología. Hospital Vall d'Hebrón. Barcelona.

Actas Urol Esp 2006; 30 (5): 513-516

\section{RESUMEN}

\section{ESTADO ACTUAL DE LA PROSTATECTOMÍA RADICAL LAPAROSCÓPICA}

La cirugía laparoscópica puede considerarse que ha alcanzado la mayoría de edad al indicarse en la patología oncológica, y debe su desarrollo a las escuelas francesas que sistematizaron su empleo en el cáncer prostático.

Cumple los principios que ha de seguir la cirugía oncológica, al igual que la cirugía convencional, pero queda aún por demostrar que los resultados a largo plazo, tanto desde el punto de vista tumoral como el funcional sean mejores que la cirugía clásica.

Cada vez son más los grupos que están incorporando estas técnicas a su quehacer diario.

Palabras clave: Laparoscopia. Prostatectomía radical.

\section{ABSTRACT \\ CURRENT STATUS OF RADICAL LAPAROSCOPIC PROSTATECTOMY}

Laparoscopic surgery can be said to have come of age when it was first indicated for cancer conditions. Advances in this field are largely due to the French school, which has made it a standard practise in prostate cancer.

It complies with the principles required for cancer as well as conventional surgery, but it remains to be verified whether its long-term results, both from tumoral and functional perspectives, are not only similar to those of classical surgery, but even better.

In fact, increasing numbers of clinical groups are incorporating this technique in their daily work. Keywords: Laparoscopy. Radical prostatectomy.

$\mathrm{L}$ a curación del cáncer, la preservación de la continencia y de la función sexual son los objetivos principales de la prostatectomía radical (PR). Esta técnica se ha demostrado como la más efectiva para el tratamiento del cáncer de próstata localizado y es el más recomendado por la mayoría de urólogos a sus pacientes. La introducción en los últimos años de la laparoscopia en la Urología y específicamente en la cirugía del cáncer de próstata, ha comportado la comparación entre la técnica clásica retropúbica (PRR) y el abordaje laparoscópico (PRL). Las primeras series ${ }^{2-4}$ que evaluaron la prostatectomía radical laparoscópica concluían que no aportaba ventajas debido a la gran dificultad técnica y la larga duración del procedimiento. Fueron Guilloneau y Vallancien ${ }^{5,6}$ , que refinaron y estandarizaron el procedimiento, aportando series cada vez más extensas con una clara mejoría de los resultados y una disminución del tiempo quirúrgico.

La alta incidencia del cáncer de próstata ha permitido que, a pesar de su dificultad técnica, la laparoscopia se haya incorporado como técnica quirúrgica para esta indicación. Esta revolución ha permitido a su vez a la Urología ir ganando nuevas indicaciones día a día. 


\section{COMPARACIÓN DE AMBAS TÉCNICAS}

Las dos técnicas quirúrgicas poseen características que les son comunes. La PRL comparte indicaciones con la PRR. El cáncer de próstata localizado es al que se dirigen todas las técnicas quirúrgicas para ofrecer sus mejores resultados. Las contraindicaciones de ambas técnicas también son compartidas, no existiendo hoy contraindicaciones absolutas específicas del abordaje laparoscópico. Por otra parte, tanto el aprendizaje como la experiencia quirúrgica son imprescindibles para una correcta ejecución de la técnica, hecho demostrado en ambos abordajes, y los resultados oncológicos y funcionales dependerán claramente de esta experiencia. Por todas estas razones, un paciente candidato a una PRR lo será también para una PRL ${ }^{7}$.

Existen a su vez claras diferencias a favor de la PRL entre los dos abordajes en diversos aspectos. La visión mediante laparoscopia es exquisita y permite nuevos horizontes en lo que se refiere al conocimiento anatómico. El sangrado se ha demostrado claramente menor en la PRL según nuestra serie. El tiempo quirúrgico, que fue un inconveniente al inicio de todas las series, se ha ido reduciendo para convertirse en competitivo con la PRR. Estas diferencias se resumen en estudios recientes (Tabla 1). El coste de la técnica es muy variable dependiendo de los requerimientos técnicos solicitados por el equipo quirúrgico, siendo superior el de la PRL en EEUU, y a la inversa en Europa (datos relativos a Francia) por el uso de instrumental inventariable. El dolor postoperatorio es menor, mientras que la estan-

Tabla 1

\begin{tabular}{ll}
\hline $\begin{array}{l}\text { Tiempo quirúrgico } \\
\text { PRA } 2 \text { horas }\end{array}$ & PRL 4,5/2,6h \\
Coste de la técnica & \\
- Francia & PRA $>$ PRL \\
- EEUU & PRL $>$ PARA \\
Estancia hospitalaria & \\
- Europa & PRL 5-7 días \\
- EEUU & PRA 2-5 días \\
Reincorporación a la vida laboral & \\
PRA 47 dias & PRL 30 dias \\
\hline
\end{tabular}

Salomon, L Open vs. laparoscopic radical prostatectomy (I) BJU Int 94:238-43 (2004) cia hospitalaria presenta resultados paradóji$\cos ^{10,11}$. Debido al sistema sanitario de distinta concepción a los dos lados del Atlántico, la estancia es superior en Europa para la PRL frente a los EEUU, con estancias inferiores en PRR. En lo referente a la reincorporación a la vida laboral, la ventaja es también para la PRL.

Debemos reconocer que la PRL se ha beneficiado obviamente de la experiencia ganada durante veinte años en la PRR, con la evolución de las distintas técnicas quirúrgicas, la posibilidad y las técnicas para preservar las bandeletas y el valor para la continencia inmediata que tiene la conservación del cuello vesical. El conocimiento del valor actual de la linfadenectomía en los pacientes candidatos a la técnica y el manejo de los márgenes positivos, son hechos todos ellos incorporados por el cirujano laparoscopista a partir de esa experiencia previa.

Por otro lado, la PRL ha ofrecido a la PRR un mayor conocimiento de la anatomía pélvica, obteniendo una detallada visión anatómica nunca vista por cirugía abierta hasta el momento. También ha propiciado un mejor control del sangrado y gracias al adelantar la retirada de la sonda, ha estimulado su retirada precoz también en la PRR.

\section{TÉCNICAS DE PROSTATECTOMÍA RADICAL LAPAROSCÓPICA}

Existen diversas técnicas de PRL, siendo los diversos cirujanos los que han ido evolucionando su particular visión de cada abordaje, no habiéndose demostrado diferencias destacadas entre ellas $^{12}$. En EEUU tiene relevancia el abordaje asistido por robot mientras que en Europa no se ha generalizado.

- Transperitoneal posterior (Guilloneau,
Gaston)
- Extraperitoneal (Dubernard, Stolzenberg,
Bollens)
- Transperitoneal anterior (Gill)
- Transperitoneal anterior retrógrada
(Rassweiler)
- Transperitoneal anterior intrafascial
(Gaston)
- Asistida por robot


Aunque no es el objeto de esta revisión el detallar la técnica quirúrgica sí que daremos una pincelada sobre las distintas variantes. La vía transperitoneal posterior se inicia con la disección de la vía seminal en el fondo de saco de Douglas, para proseguir con la disección del espacio de Retzius y la prostatectomía anterógrada. Fue la técnica descrita inicialmente y a partir de la que se desarrollaron las posteriores. La vía extraperitoneal reproduce la técnica de la prostatectomía radical anterógrada por vía preperitoneal, con la dificultad de la colocación de los trócares y el espacio angosto de trabajo, y la ventaja de no involucrar en la cirugía a los órganos intraperitoneales. Tiene utilidad clara en aquellos casos de cirugía abdominal previa. Es una técnica más rápida de acceso y reduce el tiempo quirúrgico. Esta técnica es la que utilizamos habitualmente en nuestro centro. La vía transperitoneal anterior es una técnica híbrida entre estas dos, ya que reproduce la técnica extraperitoneal pero entrando por el espacio peritoneal, lo que facilita la colocación de los trócares y permite un amplio espacio de trabajo. La vía retrógrada reproduce la técnica retrógrada descrita por Walsh que es familiar en la cirugía a cielo abierto, aunque tiene el inconveniente de la visión dificultosa desde una óptica de imagen cenital. La vía interfascial supone un nuevo refinamiento en el abordaje laparoscópico, que facilita la conservación de los haces neurovasculares y realiza la exéresis de la glándula prostática por dentro de la fascia endopélvica, sin precisar su apertura.

\section{COMPLICACIONES PEROPERATORIAS}

Actualmente no disponemos de estudios prospectivos y randomizados que comparen los diferentes abordajes quirúrgicos del cáncer de próstata localizado (retropúbico, perineal y laparoscópico), pero existen series que comparan la vía abierta con la laparoscópica con resultados significativos ${ }^{11}$. La visión magnificada que proporciona la laparoscopia junto con el efecto tampón del $\mathrm{CO}_{2}$ contribuyen a un menor sangrado venoso durante la cirugía. Además, actualmente, la conversión a cirugía abierta de una PRL es un hecho muy infrecuente en los equipos habituados. Las posibles complicaciones quirúrgicas y postoperatorias se presentan en la Tabla 2.
Tabla 2

\begin{tabular}{lcc}
\hline & PRA & PRL \\
\hline Sangrado (trasfusión) & $9 \%$ & $3 \%$ \\
Lesiones rectales, ureterales, etc. & $8-18 \%$ & $4-17 \%$ \\
Fistula urinaria & $0,3-8,6 \%$ & $4-17 \%$ \\
Estenosis anastomosis & $1-5 \%$ & $1,6-3,3 \%$ \\
\hline
\end{tabular}

Salomon, L Open vs. laparoscopic radical prostatectomy (I) BJU Int. 2004;94:238-243.

\section{RESULTADOS ONCOLÓGICOS}

Los estudios publicados en relación a resultados oncológicos no se evalúan en base a la supervivencia, ya que suele ser dilatada, si no que valoran la progresión biológica, y la tasa de márgenes quirúrgicos positivos (Tabla 3).

El seguimiento después de la PRL es todavía demasiado corto para poder establecer curvas de progresión-supervivencia, aunque estos primeros datos apuntan a resultados, como mínimo, equivalentes a otras formas de $\mathrm{PR}^{8-10}$.

\section{RESULTADOS FUNCIONALES}

Continencia: Los datos publicados son confusos de interpretar, ya que la definición de continencia varía considerablemente según los estudios, desde "no protección" a "no más de una protección”. El método de evaluación de la continencia también varía, pudiendo ser realizada por el cirujano vs un médico independiente vs un cuestionario rellenado por el propio paciente. Independientemente de estos conceptos, los diferentes estudios muestran resultados similares. Así en la PRR, más de 95\% de pacientes son continentes, mientras que las tasas iniciales de la PRL después de menos de 1 año de seguimiento son del $85-97 \%^{11}$.

Tabla 3

\begin{tabular}{lcc}
\hline & PRA & PRL \\
\hline Implantes tumorales & $0 \%$ & $0 \%$ \\
Márgenes quirúrgicos & $28 \%$ & $19-23 \%$ \\
Localización & Ápex & Posterolat \\
PSA < 0,1 ng/ml (3 años) & $93 \%$ & $91,5 \%$ \\
\hline
\end{tabular}

Salomon, L Open vs. laparoscopic radical prostatectomy (II) BJU Int 2004;94:244-250. 
Erección: Nuevamente existen muchas disparidades en la definición de consenso. La definición de potencia sexual difiere según el criterio usado de erección, con o sin capacidad de penetración. Muchas veces no se especifica si el paciente usa tratamientos para la impotencia del tipo de inhibidores de la PDE. Además, la mayoría de series están de acuerdo en que se requiere un mínimo de 18 meses de seguimiento para evaluar una disfunción eréctil ${ }^{13,14}$. Teniendo en cuenta estos déficits, los resultados referentes a la tasa de impotencia cuando se preservan las bandeletas bilateralmente son del $31,9-86 \%$ para PRR y del 40-75\% para PRL ${ }^{13,15}$.

\section{APRENDIZAJE}

Cuando Walsh se preguntó en 1994 cuántas PRR debe realizar un cirujano hasta convertirse en completamente operativo, él mismo se respondió: "Tantas como sea posible"16. El mismo principio se cumple para la PRL. Por esto, es importante destacar varios principios fundamentales para iniciarse en la técnica: la PRL es una técnica compleja, hecho reconocido por los propios expertos en laparoscopia $^{17}$. El mejor método para aprender es, después de tener unas bases sentadas de hábito laparoscópico experimental, mediante tutores experimentados $^{18}$ y es necesaria una actividad laparoscópica reiterada para un buen desarrollo técnico $(>50)^{19}$. Teniendo en cuenta estas premisas, cualquier equipo que cumpla con las características descritas debería poder iniciarse y evolucionar en la cirugía laparoscópica.

\section{CONCLUSIONES}

En el momento actual, ninguno de los abordajes quirúrgicos disponibles parece ser claramente superior al resto. Los resultados de la PR son "cirujano-dependientes" y la selección de pacientes tiene una considerable influencia en la indicación, independientemente al abordaje quirúrgico escogido. Aunque las ventajas de la PRL todavía no han estado claramente establecidas y la técnica de referencia sigue siendo la PRR, la PRL está ganando terreno, con resultados, como mínimo, comparables, con menor pérdida sanguínea y más rápida recuperación postquirúrgica. En nuestra opinión, la PRL posee ventajas que hacen prever mejores resultados en continencia y recuperación de la erección aunque no se hayan podido demostrar en las series publicadas.
Probablemente, en el futuro próximo la discusión no será la competencia entre las distintas técnicas de cirugía si no con las nuevas opciones terapéuticas no quirúrgicas como la radioterapia, la braquiterapia, la crioterapia o la HIFU.

\section{REFERENCIAS}

1. Catalona WJ, Carvalhal GF, Mager DE, Smith DS. Potency, continence and complication rates in 1,870 consecutive radical retropubic prostatectomies. J Urol. 1999 Aug; 162(2):433-438.

2. Schuessler WW, Kavoussi LR, Clayman RV, Vancaille TH. Laparoscopic radical prostatectomy: initial case report. J Urol. 1992;147:2463.

3. Raboy A, Ferzli G, Albert P. Initial experience with extraperitoneal endoscopic radical retropubic prostatectomy. Urology. 1997 Dec;50(6):849-853. Review.

4. Guillonneau B, Cathelineau X, Doublet. JD et al. Prospective assessment of functional results after laparoscopic radical prostatectomy. J Urol. suppl 2001;165 (Suppl.5):150, Abs 614

5. Guillonneau B, Vallancien G. Laparoscopic radical prostatectomy: the Montsouris experience. J Urol. 2000 Feb;163(2):418-422.

6. Guillonneau B, Vallancien G. Laparoscopic radical prostatectomy: oncological evaluation after 1,000 cases a Montsouris Institute. J Urol. 2003 Apr;169(4): 1261-1266.

7. Hasan WA, Gill IS. Laparoscopic radical prostatectomy: current status. BJU Int 2004;94:7-11.

8. Guilloneau B, El-Fettouh H, Baumert $\mathrm{H}$ et al. Laparoscopic radical prostatectomy: oncological evaluation after 1,000 cases a Montsouris Institute. J Urol 2003 Apr;169(4):1261-1266.

9. Salomon L, Levrel O, Anastasiadis AG, Saint F, De La Taille A, Cicco A, et al. Outcome and complications of radical prostatectomy in patients with PSA $<10$ $\mathrm{ng} / \mathrm{ml}$ : comparison between the retropubic, perineal and laparoscopic approach. Prostate Cancer Prostatic Dis. 2002;5(4):285-290.

10. Salomon L, Levrel O, De la Taille A, Anastasiadis AG, Saint F, Zaki S, et al. Radical prostatectomy by the retropubic, perineal and laparoscopic approach: 12 years of experience in one center. Eur Urol. 2002 Aug;42(2):104-10; discussion 110-111.

11. Salomon L, Sebe P, De la Taille A, Vords D, Hoznek A, Yiou R, et al. Open versus laparoscopic radical prostatectomy: Part II. BJU Int. 2004 Jul;94(2):244250. Review.

12. Eden CG, King D, Kooiman GG, Adams TH, Sullivan ME, Vass JA. Transperitoneal or extraperitoneal laparoscopic radical prostatectomy: Does the approach matter? J Urol. 1004 Des;172:2218-2223.

13. Walsh PC. Patient-reported urinary continence and sexual function after anatomic radical prostatectomy. Urology. 2000 Jan;55(1):58-61.

14. Kim ED, Nath R, Slawin KM, Kadmon D, Miles BJ, Scardino PT. Bilateral nerve grafting during radical retropubic prostatectomy: extended follow-up. Urology. 2001 Dec;58(6):983-987.

15. Vallancien G, Guillonneau B, Cathelineau X, Baumert H, Doublet JD. Localized prostatic cancer: treatment with laparoscopic radical prostatectomy: study with 841 cases. Bull Acad Natl Med. 2002;186(1):117-123; discussion 123-124. French.

16. Walsh PC. The status of radical prostatectomy in the United States in 1993: where do we go from here? J Urol. 1994 Nov;152(5 Pt 2):1816.

17. Guillonneau B, Abbou CC, Doublet JD, Gaston R, Janetschek G, Mandressi A, et al. Proposal for a "European Scoring System for Laparoscopic Operations in Urology”. Eur Urol. 2001 Jul;40(1):2-6; discussion 7.

18. Fabrizio MD Laparoscopic radical prostatectomy: decreasing the learning curve using a mentor initiated approach. J Urol. 2003 Jun;169(6):2063-2065.

19. ElFeel A, Davis JW, Deger S, et al. Laparoscopic radical prostatectomy-an analysis of factors affecting operating time. Urology. 2003 Aug;62(2):314-318.

Dr. L. Cecchini Rosell

E-mail: lcecchini@vhebron.net 